\title{
Optical lenses and magnification in archery*
}

\section{B Strydom and JT Ferreira}

\author{
Department of Optometry, University of Johannesburg, PO Box 524, Auckland Park, 2006 South \\ Africa
}

<jferreira@uj.ac.za>

Received 25 November 2009; revised version accepted 5 March 2010

\begin{abstract}
The use of optical lenses to increase or magnify a target of concern is commonly used in compound bow archery. The principles and factors that may influence the use of these lenses may, however, not be fully understood by archers. Two types of lens systems, the one-lens system and the two-lens system, as described by the authors, may be used. Different factors such as the draw length of the archer,
\end{abstract}

the ametropic condition of the archer, depth of field, depth of focus, age, accommodation, the power of $F_{1}$ and $F_{2}$ (see Figures 1 and 2 in the paper) and the distance the lens is placed from the archer may influence the results of the lens used by the archer. (S Afr Optom 2010 69(1) 29-34)

Key words: optical lens, magnification, compound bow archery, one- and two-lens systems in archery.

\section{Introduction}

Optical lenses of positive power are used to magnify the image of objects. Magnification is specifically used in archery to enlarge the target image and to obtain greater detail on a desired target ${ }^{1}$. Depending on many factors, such magnification may be sufficient and/or may be accompanied by undesirable factors such as target blur. A telescope consists out of two lenses, separated by a distance d, that magnify distance objects. A telescope is afocal when the light enters and leaves the system parallel. The Galilean telescope has a diverging ocular lens and a converging objective lens. The image obtained through a Galilean telescope is magnified and erect ${ }^{1,2}$. When using a Keplerian telescope both the objective lens and the ocular lens are converging lenses and produces a magnified but inverted image ${ }^{2}$. In archery, especially compound bow shooting, some archers use optical aids, thus a magnifying lens to enlarge the image of the target. This is a very confusing field as the archer does not definitely know what lens system would be the best for him or her. The lenses used are too expensive to use on a trial and error system.

\section{The bow sight}

The sight on a compound bow usually comprises of a circular ring with a pin. The top edge of the pin is attached to an optical fibre to form a pin head. When aiming the archer aligns the peep sight with the pin head, and then aligns this system with the target. The peep sight is situated in the string of a bow, at a specific level so that the archer can look through it and this creates a pinhole effect. Different types of sights are available, for example a five pin sight has five pins that can be aligned in such a way that the archer can shoot distances ranging from 10 metres up to, say 50 . 
The archer would achieve this by aligning the desired pin with the target for a specific distance ${ }^{3}$. There are also sights that use only one pin that is attached to a "slider arm" making adjustments for height for different shooting distances. Normally there are adjustment knobs on the sight allowing for lateral and vertical adjustments and an extension bar that can be used to vary the distance of the bow sight that is normally at arm's length from the archer ${ }^{4}$.

In traditional archery no sights are used. In recurve bow archery the use of lenses or any other optical or electronic aids (for example, a pinhole), helping the archer to aim better, is disallowed ${ }^{4}$. In compound archery optical aids may play an important role and many of the best compound archers do use such lenses ${ }^{1}$. Tournament structures in compound bow archery allow the archer to compete at top level with lenses as an optical aid. This would be in a specific shooting category (freestyle), where archers with any optical aids are accommodated ${ }^{5}$. Although Long and Haywood ${ }^{4}$ did investigate the optical characteristics of lenses used in archery, not much research has been done to specifically investigate the use of lenses and magnification in the sport ${ }^{4}$. Our study aims to simplify and clarify the use of lenses in the sport of archery and to identify and discuss the factors that may influence the performance of the archer when using these lenses.

Two types of lens systems (one and two lens systems) may be important:

\section{The one-lens system}

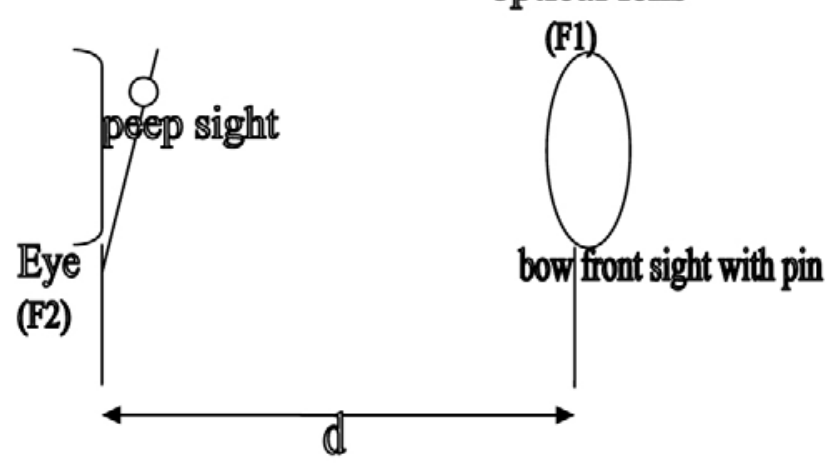

Figure 1. Illustration of the one lens system

With the one lens system, the archer only uses one lens $\left(\mathrm{F}_{1}\right)$, and this lens is placed in the front bow sight plane. The uncompensated refractive error of the eye, in the ocular plane, would be $\mathrm{F}_{2}$ (see Figure $1)$. The uncompensated refractive error would then act as the second lens $\left(\mathrm{F}_{2}\right)$. The distance between $\mathrm{F}_{1}$ and $\mathrm{F}_{2}$ would be (d) in metres. Long and Haywood (1990) did a study ${ }^{4}$ to explain the optical characteristics of aiming scopes (usage of lenses) in archery. They concluded that lenses used in archery are simple converging lenses with powers up to $1 \mathrm{D}$. The problem they had was that with the use of these lenses, the stability of the archer was important as the lens power increased a reduction in visual acuity (blur) of the target occurred ${ }^{4}$. The following equations may be used to determine the appropriate variables 5,6 :

$d=1 / F_{1}+1 / F_{s}$

and

$\mathrm{M}=-\mathrm{F}_{\mathrm{s}} / \mathrm{F}_{1}$

where $\mathrm{M}=$ Magnification, $\mathrm{d}=$ the distance between the lens and the eye (m), $F_{1}=$ power of lens (D) placed at distance $d$ and $F_{s}=F_{2}=$ the refractive error of the eye (D).

\section{The two-lens system}

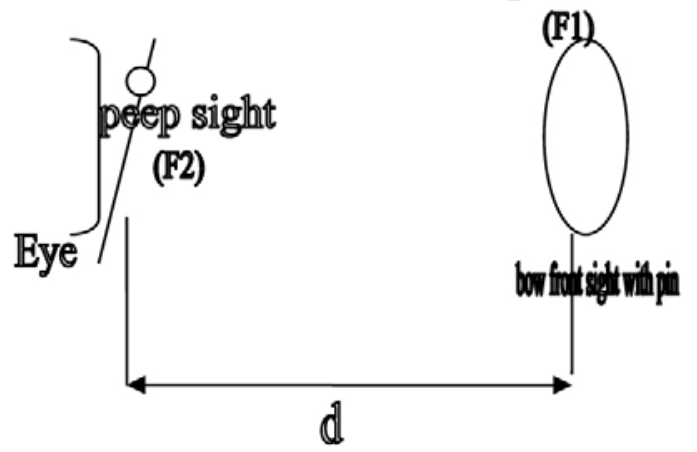

Figure 2. Illustration of the two lens system

In the two lens system, one lens $\left(F_{1}\right)$ is situated in the bow front sight plane and the second lens $\left(\mathrm{F}_{2}\right)$ is situated in the peep sight plane, the latter being close to the eye and coinciding with the spectacle plane. The light will enter and exit this system parallel and no accommodation would be required. Let us refer to the powers of these lenses as $F_{1}$ and $F_{2}$ respectively. Usually the lens in the bow front sight plane (the objective lens $\left(F_{1}\right)$ ) will be chosen and the distance $(d)$ between $F_{1}$ and $F_{2}$ will be specific according to the archer's draw length. Using the vergence of light, the 
power of the lens in the peep sight plane (ocular lens $\left(\mathrm{F}_{2}\right)$ ) can be obtained 6 . When using the two lens system, the power of $F_{1}$ would be chosen, the distance $\mathrm{d}$ would depend on the archers draw length and $\mathrm{F}_{2}$ calculated by the following equations 5,6 :

$\mathrm{M}=-\mathrm{F}_{2} / \mathrm{F}_{1}$

where

$\mathrm{M}=$ Magnification, $\mathrm{F}_{1}=$ Objective lens and $\mathrm{F}_{2}=$ Ocular lens and

$\mathrm{d}=1 / \mathrm{F}_{1}+1 / \mathrm{F}_{2}$

where $\mathrm{d}=$ the distance from the peephole plane to the plane of the lens used in the bow front sight (metres), $\mathrm{F}_{1}=$ power of the objective lens (Dioptres) and $\mathrm{F}_{2}=$ power of the ocular lens (Dioptres).

If the magnification is negative then the image will be inverted and if magnification is positive then the image will be upright. Also if the magnification is greater than 1, then the object is magnified. However, if magnification is less than 1 the object is minified. As the draw length is dependant on the length of the arms of the archer, the refractive error is an important factor to consider when using the one lens system.

The possible effect of ametropia on the one-lens system

\section{Emmetropia}

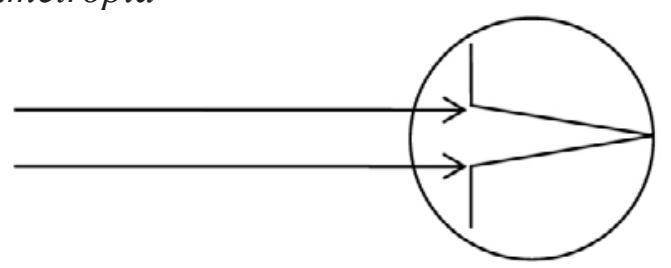

Figure 3. The emmetropic eye

As discussed previously, emmetropia, is the normal refractive condition of the eye. When an emmetrope does not use any accommodation, parallel light rays would converge to a sharp focus on the retina $^{7}$ (see Figure 3). According to equation 2, an emmetrope $(0 \mathrm{D})$ would get magnification of 0 . Although this may be true in theory, accommodation is an important factor with the pre-presbyopic individual. It may be true for an older individual with no accommodation, (absolute presbyope), or a younger person with an insufficient accommodation system. However, this may not be the case for an individual that can still accommodate. In the case of an emmetrope, or an ametropic archer with his or her refractive error corrected, target clarity would be influenced by many factors. Factors such as the size of the peep sight, the amount of magnification needed, the power of the lens used, the draw length of the archer and age may influence the end result ${ }^{6}$.

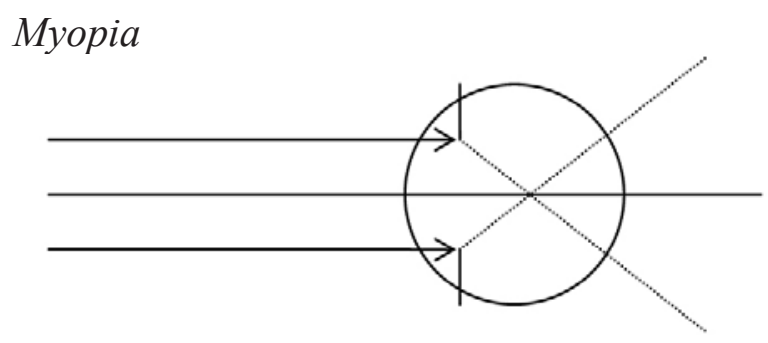

Figure 4. The myopic eye

The myopic eye with accommodation relaxed, is defined as the ametropic condition where parallel light converges to a point in front of the retina (See Figure 4). An ametropic eye does not, as with emmetropia, form a point image on the retina, but forms a blur circle on the retina. For myopia, the size of the blur circle would depend on the amount of myopia and the pupil size ${ }^{7}$. This means for a $1 \mathrm{D}$ myopic archer the objective lens that must be held up at $0.80 \mathrm{~m}$ (in the bow front sight plane), should be $-5 \mathrm{D}$. This also means that there would be minification of 0.20 . Lastly the image is upright, but this may not be the case for all uncorrected myopes. In summary, it may not be beneficial for an archer with a significant myopic refractive error to use a single lens for magnification. The solution may be to correct the myopic refractive error and calculate the optimal lens for magnification when using this correction.

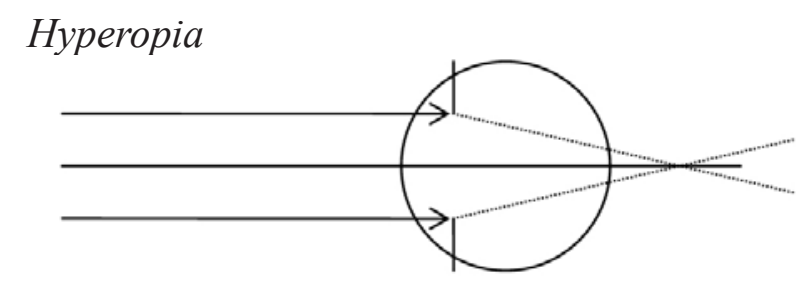

(a)

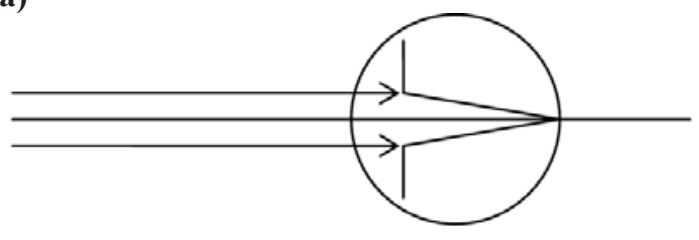

(b)

Figure 5. The hyperopic eye: (a) Viewing an object at a distance, with accommodation relaxed. (b) Viewing an object at a distance, with accommodation in place. 
Hyperopia, is the ametropic condition when parallel light, (with accommodation relaxed) converge to focus behind the retina. The result would then be a blur circle on the retina (see figure 5(a)). However, with accommodation taking place, the size of the blur circle on the retina (when viewing distance objects) may be greatly reduced, depending on the age and amplitude of accommodation (see figure 5(b)). Near visual acuity of a hyperope will depend on the distance of the object, the amplitude of accommodation and the amount of hyperopia present ${ }^{7}$. From equations 1 and 2 this means that for a $1 \mathrm{D}$ hyperopic archer, the lens that must be held up at $0.80 \mathrm{~m}$, would be +0.50 D. This also means that there would be an upright image, with magnification of 2 . The quality of the image would be dependant on factors such as the position of the blur circle regarding the retina, depth of focus and the size of the peep sight. It may also depend on accommodation ability. For a pre-presbyopic hyperopic archer that has an efficient accommodation system, it may be possible to use this lens with his uncorrected refractive error. For an archer that does not have an efficient accommodation system, or a presbyopic archer, it may be difficult to use the lens mentioned in the example. Again it would depend on the preference of the individual regarding the quality of the image. The refractive error plays a major part in the clarity of the target when using the one-lens system, as discussed earlier. When using a single lens to magnify an object, it may be suitable to compensate for the total refractive error of an archer if he is significantly myopic or astigmatic. If choosing to correct the ametropia, the choice between contact lens or spectacle correction, single vision or multifocal correction and the advantage and disadvantage of each must be discussed with the archer. The end result must be beneficial and satisfactory to the archer, without being a limiting factor to their sporting performance.

Factors to consider when using the one-lens system Pupil size

Long and Haywood ${ }^{4}$ suggested that using a smaller peep sight is beneficial. If the archer uses his or her fingers, it may result in torque of the string and resultant peep sight misalignment, The torque of the string is minimal with a mechanical trigger. Different size peep sights are available. Thus, an archer should use the smallest peep sight that still allows alignment and visibility of the bow sight and target. According to Grosvenor ${ }^{7}$, the optimum ocular pupil size for the best visual acuity is between 2 and $5 \mathrm{~mm}$, anything smaller and the visual acuity may be reduced due to diffraction effects. Pupil size greater than $5 \mathrm{~mm}$, may cause spherical aberration that would reduce the visual acuity and cause blur 7 .

\section{Depth of field and depth of focus}

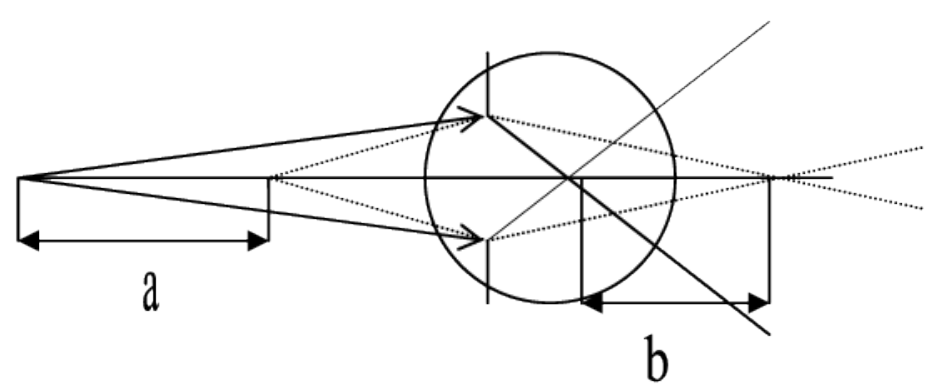

Figure 6. (a) Depth of field of the eye, (b) Depth of focus of the eye

The retinal image of an object, perceived by an individual may be seen clear, without the image being sharply focused on the retina. This is due to the depth of focus of the eye (see Figure 6 (b)). Depth of focus is the range in front or behind the retina where light may form an image and still be seen clearly ${ }^{7}$. The depth of field would then be the area in object space in which an individual can move an object closer or away from him or herself, and still see this object clearly. However, distinguishing between these two areas may be difficult in pre-presbyopic individuals due to the accommodation system. It may, however, be easier illustrated in those that can no longer accommodate (absolute presbyope) ${ }^{7}$.

\section{Age and accommodation}

The size of the blur circle, situated on the retina and present with ametropic conditions such as myopia and hyperopia, may be changed when accommodation takes place 7 . This will depend on factors such as the amount and type of ametropia, the amplitude of accommodation and the age of the subject. The age of the archer plays a role as presbyopia become more evident with aging persons, starting at more or less the age of 40 years 7 . 
Distance of lens held up (d) before the eye

Although the draw length of an archer is relatively fixed, the distance between the eye and the lens $\left(\mathrm{F}_{1}\right)$ used as a magnifier, can be changed via an extension bar $^{3}$. An archer that does not want to change his or her draw length, but still needs more magnification, may benefit by using such an extension bar ${ }^{4}$.

\section{Factors to consider when using the two-lens system}

Power of $F_{1}$

The important factor here is to understand the role of each lens. Due to the differing of the draw lengths, the power of $F_{1}$ must be chosen carefully for each archer. Choosing the power of $F_{1}$ too high for an archer with a shorter draw length, may result in excessivemagnification and thus inconsistency when shooting.

According to Long and Haywood ${ }^{4}$ there is a relationship between successful archers and body stability, and many novice archers find high powered scopes difficult to use. If the power of $F_{1}$ is chosen too high, a high amount of magnification may be the result. This high amount of magnification may influence the archer's consistency due to the difficulty of alignment between the archer and the target increasing as the magnification increases.

\section{Power of $\mathrm{F}_{2}$}

The power of $\mathrm{F}_{2}$ depends on the draw length of the archer and the power of $F_{1}$. The greater the power of $F_{1}$ and the shorter the draw length of an archer, the higher the power of $F_{2}$ becomes. An example would be an archer with a 29 inch draw length, having a distance (d) between $F_{1}$ and $F_{2}$ of more or less $0.80 \mathrm{~m}$. When choosing the front sight lens power $\left(\mathrm{F}_{1}\right)$ to be $+2 \mathrm{D}$, according to equation 4 , the power of $\mathrm{F}_{2}$ would be $3.33 \mathrm{D}$. According to equation 3 this would result in magnification of -1.67 . However an archer with a drawlength of 24 inches would have a distance (d) between $F_{1}$ and $F_{2}$ of more or less $0.4 \mathrm{~m}$. If the front sight lens power $\left(\mathrm{F}_{1}\right)$ is $+2 \mathrm{D}$ again then according to equation 4 the power of $\mathrm{F}_{2}$ would be $-10 \mathrm{D}$. According to equation 3 the resultant magnification would be 5. This may not be the optimum results for aforementioned archers.

\section{Draw length}

If an archer has a shorter draw length, a higher powered lens may be more appropriate for the desired magnification needed. Thus, a greater draw length may need a lower dioptric powered lens in the front sight $\left(\mathrm{F}_{1}\right)$, and a shorter draw length, may need a higher dioptric powered front sight lens $\left(\mathrm{F}_{1}\right)$, for optimum magnification. This may also depend on the experience of the archer as a more experienced archer, being more stable in shooting form, may find it easier to use a high powered lens.

\section{Peep sight}

The size of the ocular pupil may be reduced through increasing the illumination ${ }^{7}$, or by using a pinhole. The peep-sight can act as a pinhole. A person with presbyopia that may find it easier to shoot in bright sunlight due to ocular pupil constriction, and thus retinal blur circle reduction as well as an increased depth of field. This may be a useful way of increasing depth of field by reducing the peep sight diameter to decrease the size of the retinal blur circle. If the refractive error is corrected, then the optimal pupil size would be between $2 \mathrm{~mm}$ and $5 \mathrm{~mm}$ for the best visual acuity, as pupil size smaller than $2 \mathrm{~mm}$ would reduce visual acuity due to diffraction effects. If the pupil size is greater than $5 \mathrm{~mm}$ the effect of spherical aberration may too reduce the visual acuity $^{7}$. Choosing a smaller diameter peep sight may help to reduce spherical aberrations and may be useful in giving more clarity of the object of interest. This blur minimization is also called the pinhole effect ${ }^{2}$. The effect of ocular pupil size on visual acuity depends on the correction of the eye's refractive error. If the refractive error is compensated, then the optical image formed by an object will not coincide with the retina and a blurred retinal image may be the result depending on the type of ametropia and the state of accommodation. The size of the retinal blur circle for this object would increase with an increase of the distance of the optical image from the retina as well as with pupil size. This means that if pupil size is reduced for an uncorrected refractive error, then the visual acuity would be increased ${ }^{7}$.

\section{Ametropia}

When the optical lenses $\left(F_{1}\right.$ and $\left.F_{2}\right)$ are chosen, the existing ametropia must be compensated where needed. If the archer wants the target to be clear, then the optimal correction of a high myopic archer must 
be considered. It may or may not be necessary to correct a hyperopic archer. Age does play a role in this consideration and if archers are pre-presbyopic, then uncompensated hyperopes (to a certain amount of hyperopia) would find it much easier to view the target clearly through the two-lens system than a significant uncompensated myopic archer. If the archer is presbyopic, then the distance compensation of the hyperopic or myopic archer must be considered and used during archery. However, this may be true for an archer that wants the target to be clear. If the archer would like the pins to be clear, then this must be noted and may the myopic archer be left slightly uncompensated to enable clarity of the sight plane. The uncompensated hyperopic archer may need a part of his near compensation. Again, as with the one-lens system, the consideration must be according to what are the archer needs, and may involve partial compensation of the distance and or near refraction with spectacles or contact lenses, single vision or multifocal compensation. This suggests that archers requiring magnifying lenses, should be compensated with their optimal distance compensating prescription, as magnification of the target is the main goal when using this lenses.

\section{Conclusion}

Communicating with the archer to identify the area of importance as well as clarity with regards to the sight plane or target plane and discussing the advantages and disadvantages of each possible choice may aid in choosing the correct lens system. When choosing lenses $\left(\mathrm{F}_{1}\right.$ and/or $\left.\mathrm{F}_{2}\right)$ to create magnification of a desired target, the archer's age, refractive status, draw length, ocular pupil size, peep sight size, accommodation ability and the required magnification should be kept in mind. It is also important to understand that with greater magnification, the stability of the archer would be less, leading to a reduction of target clarity and archery experience must also be considered. Preference regarding the one- or two-lens systems, must also be noted and respected.

\section{References}

1. Personal communication with Herholdt F, Krugersdorp, 2007.

2. Keating MP. Geometric, Physical and Visual Optics. London: Butterworths, 1988.

3. Schuh D. Bowhunters Encyclopedia. USA: Stackpole Books, 1987.

4. Long WS, Haywood KS. The optical characteristics of aiming scopes in archery. Journal of the American Optometric Association 199061 777-781.

5. Herholdt F. Target Archery. Africa's Bowhunter and Archer Magazine 2007 8(4) 45.

6. Personal communication with Harris WF, University of Johannesburg, June 2008.

7. Grosvenor T. Primary Care Optometry, $4^{\text {th }}$ Edition. Oxford: Butterworth and Heinemann, 2002. 